\title{
Significance of albumin to globulin ratio as a predictor of febrile urinary tract infection after ureteroscopic lithotripsy
}

\author{
Seung Yun Yi ${ }^{1}$, Dong Jin Park ${ }^{2}$, Kyungchan Min ${ }^{1}$, Jae-Wook Chung ${ }^{3}$, Yun-Sok Ha ${ }^{3}$, Bum Soo Kim ${ }^{4}$, \\ Hyun Tae Kim ${ }^{4}$, Tae-Hwan Kim ${ }^{3}$, Eun Sang Yoo ${ }^{4}$ \\ ${ }^{1}$ Department of Urology, Kyungpook National University Hospital, Daegu, Korea \\ ${ }^{2}$ Department of Urology, Dongguk University Gyeonju Hospital, Gyeongju, Korea \\ ${ }^{3}$ Department of Urology, School of Medicine, Kyungpook National University, Kyungpook National University Chilgok Hospital, Daegu, Korea \\ ${ }^{4}$ Department of Urology, School of Medicine, Kyungpook National University, Kyungpook National University Hospital, Daegu, Korea
}

Received: January 31, 2021

Revised: March 18, 2021

Accepted: March 19, 2021

Corresponding author:

Eun Sang Yoo, MD

Department of Urology, School of

Medicine, Kyungpook National

University, Kyungpook National

University Hospital, 680

Gukchaebosang-ro, Jung-gu, Daegu

41944, Korea

Tel: $+82-53-420-5843$

Fax: +82-53-421-9618

E-mail: uroyoo@knu.ac.kr
Background: We aimed to analyze the effectiveness of albumin to globulin ratio (AGR) in predicting postoperative febrile urinary tract infection (fUTI) after ureteroscopic lithotripsy (URS) and retrograde intrarenal surgery (RIRS).

Methods: From January 2013 to May 2018, 332 patients underwent URS and RIRS. The rate of postoperative fUTI and risk factors for postoperative fUTI were analyzed using logistic regression. Patients were divided into postoperative fUTI and non-postoperative fUTI (non-fUTI) groups. AGR with other demographic and perioperative data were compared between the two groups to predict the development of fUTI after URS.

Results: Of the 332 patients, postoperative fUTI occurred in 41 (12.3\%). Preoperative pyuria, microscopic hematuria, diabetes mellitus, hypoalbuminemia, and hyperglobulinemia were more prevalent in the fUTI group. Patients in the fUTI group had larger stone size, lower preoperative $A G R$, longer operation time, and longer preoperative antibiotic coverage period. In a multivariable logistic analysis, preoperative pyuria, $A G R$, and stone size were independently correlated with postoperative fUTI $(p<0.001, p=0.008$, and $p=0.041$, respectively). Receiver operating curve analysis showed that the cutoff value of AGR that could predict a high risk of fUTI after URS was 1.437 (sensitivity, 77.3\%; specificity, 76.9\%), while the cutoff value of stone size was $8.5 \mathrm{~mm}$ (sensitivity, 55.3\%; specificity, 44.7\%).

Conclusion: This study demonstrated that preoperative pyuria, AGR, and stone size can serve as prognostic factors for predicting fUTI after URS.

Keywords: Albumin to globulin ratio; Biomarkers; Nephrolithiasis; Ureteroscopy; Urinary tract infections

\section{Introduction}

Advancements in endourology have introduced the era of retrograde intrarenal surgery (RIRS) and ureteroscopic lithotripsy
(URS), with a higher stone-free rate and lower morbidity [1]. However, postoperative urinary tract infection (UTI) and subsequent sepsis remain a burden for postoperative care, despite proper administration of prophylactic intravenous antibiotics. UTI is one

Copyright (C) 2021 Yeungnam University College of Medicine

This is an Open Access article distributed under the terms of the Creative Commons Attribution Non-Commercial License (http://creativecommons.org/licenses/by-nc/4.0/) which permits unrestricted non-commercial use, distribution, and reproduction in any medium, provided the original work is properly cited. 
of the most common postoperative complications after RIRS, and it has been reported that the rate varies widely between studies [2]. Postoperative UTI can be life-threatening because it can lead to sepsis and septic shock. If it advances to septic shock, higher postoperative mortality and longer hospital stays would be expected [3]. Attempts have been made to determine the risk factors to minimize postoperative febrile UTI. Some studies have suggested that the neutrophil-to-lymphocyte ratio (NLR) and platelet-to-lymphocyte ratio (PLR) can be effective biomarkers for predicting postoperative UTI after percutaneous nephrolithotomy $[4,5]$.

Albumin and globulin are the main components of serum proteins, and these proteins play a pivotal role in acute inflammatory reactions and chronic inflammation. In this study, we validated the effectiveness of the albumin to globulin ratio (AGR) in predicting postoperative febrile UTI (fUTI). However, to date, few studies have evaluated AGR as a predictor of postoperative infection after URS and RIRS. AGR has already been studied in different types of cancer, and it has been found to be a possible prognostic marker for cancer [6]. However, so far, there have been limited studies investigating whether AGR can be used as a prognostic marker to predict postoperative fUTI in URS and RIRS.

\section{Materials and methods}

Patients who underwent URS and RIRS from January 2013 to May 2018 in a tertiary general hospital in South Korea were included in this study. Patients with bilateral stones and those without preoperative serum albumin and globulin data were excluded from this study. A total of 332 patients were included in the study. Patients were divided into the postoperative fUTI group (fUTI group) and non-postoperative fUTI group (non-fUTI group). Postoperative fUTI was diagnosed if the patient's body temperature was above $38^{\circ} \mathrm{C}$ with pyuria and other fever foci that should have been excluded within a week after the surgery. Preoperative clinical data including age, sex, and underlying diseases such as hypertension, diabetes mellitus (DM), chronic renal failure (CRF) defined as an estimated glomerular filtration rate of less than 60 $\mathrm{mL} / \mathrm{min}$ per $1.73 \mathrm{~m}^{2}$, body mass index (BMI), presence of pyuria, presence of microscopic hematuria, presence of preoperative ureteral stent and percutaneous nephrostomy (PCN), and history of previous URS and RIRS were collected. Stone size prior to surgery was measured using noncontrast computed tomography, and the largest diameter was recorded. Preoperative pyuria was defined as more than five to 10 white blood cells (WBC) per high-power field (HPF), while preoperative microscopic hematuria was defined as more than three red blood cells per HPF on laboratory urinalysis. Blood samples were collected within 1 month prior to surgery, and
AGR was calculated using the equation of 'AG ratio $=$ albumin/total protein without albumin. WBC counts were collected to demonstrate their effectiveness as an inflammatory indicator. Sterile preoperative urine culture was performed before surgery.

The patients were admitted to the hospital a day before surgery. None of the patients had preoperative fever. Fluoroquinolone was administered from the day of admission to the day of surgery. Under spinal or general anesthesia, we used a semi-rigid ureteroscope and/or flexible ureteroscope for lithotripsy. A hydrophilic guidewire was used to engage the semi-rigid ureteroscope into the ureter. For renal stones, we used Amplatz Super Stiff guidewire (Cook Medical Inc., Bloomington, IN, USA) and Flexor Ureteral Access Sheath (Cook Medical Inc.) prior to the engagement of the flexible ureteroscope. A 200- or 365-micro holmium laser lithotripter was used to fragment the target stone into pieces, with a power of 20 joules and 0.5 frequency. We used a stone basket to remove the fragments larger than $2 \mathrm{~mm}$., and six French ureteral stents were kept for 2 weeks after the surgery. Patients were discharged on postoperative day one, and antibiotics were prescribed for 5 days if there was no evidence of postoperative UTI.

Chi-square test for categorical variables and Student t-test for continuous variables were used to analyze the baseline characteristics. Multivariate logistic regression analysis was performed using the forward-likelihood ratio method, and $p$-values, odds ratios (ORs), and 95\% confidence intervals (CIs) were collected. A receiver operating characteristic (ROC) curve was used to determine the optimal cutoff value and its sensitivity and specificity. Statistical significance was set at $p<0.05$. IBM SPSS version 18.0 for Windows (IBM Corp., Armonk, NY, USA) was used for the analysis.

\section{Results}

Table 1 shows the baseline characteristics of the patients and the chi-square test between the two groups. Of the 332 patients, 41 were included in the fUTI group and 291 were included in the non-fUTI group. Of all the patients, 135 had hypertension, 70 had $\mathrm{DM}$, and nine had CRF. The mean age was $56.9 \pm 13.3$ years, mean stone size was $10.1 \pm 4.2 \mathrm{~mm}$, and mean preoperative AGR was $1.64 \pm 0.43$. Preoperative pyuria, microscopic hematuria, hypoalbuminemia, hyperglobulinemia, and DM were more prevalent in the fUTI group $(p=0.001, p=0.004, p=0.001, p=0.041$, and $p=0.037$, respectively). Patients in the fUTI group had larger stone size, lower preoperative AGR, longer operation time, and longer preoperative antibiotic coverage period $(p=0.004, p=0.003$, $p=0.028$, and $p=0.003$, respectively). There were no statistically significant differences in hypertension, CRF, age, BMI, operation histo- 
Table 1. Comparison of demographic, clinical, and postoperative data between postoperative fUTI group and non-fUTI group

\begin{tabular}{|c|c|c|c|c|}
\hline \multirow{2}{*}{ Variable } & \multirow{2}{*}{ Total } & \multicolumn{2}{|c|}{ Postoperative fUTI } & \multirow{2}{*}{$p$-value } \\
\hline & & Yes & No & \\
\hline Patient & $332(100)$ & $41(12.3)$ & $291(87.7)$ & \\
\hline Age (yr) & $56.9 \pm 13.3$ & $57.2 \pm 12.0$ & $56.9 \pm 13.3$ & 0.869 \\
\hline Sex & & & & 0.128 \\
\hline Male & $227(68.4)$ & $32(78.0)$ & $195(67.0)$ & \\
\hline Female & $105(31.6)$ & $9(22.0)$ & $96(33.0)$ & \\
\hline Hypertension & $135(40.7)$ & $17(41.5)$ & $118(40.5)$ & 0.979 \\
\hline Diabetes mellitus & $70(21.1)$ & $14(34.1)$ & $56(19.2)$ & 0.037 \\
\hline Chronic renal failure & $9(2.7)$ & $1(2.4)$ & $8(2.7)$ & 0.888 \\
\hline History of stone operation & $59(17.8)$ & $9(22.0)$ & $50(17.2)$ & 0.507 \\
\hline Body mass index $\left(\mathrm{kg} / \mathrm{m}^{2}\right)$ & $25.3 \pm 4.0$ & $25.6 \pm 5.1$ & $25.3 \pm 3.9$ & 0.688 \\
\hline Procedure & & & & 0.445 \\
\hline URS & $131(39.5)$ & $18(43.9)$ & $113(38.8)$ & \\
\hline RIRS & $180(54.2)$ & $22(53.7)$ & $158(54.3)$ & \\
\hline URS+RIRS & $21(6.3)$ & $1(2.4)$ & $20(6.9)$ & \\
\hline Preoperative pyuria & $54(16.3)$ & $9(22.0)$ & $45(15.5)$ & $<0.001$ \\
\hline Preoperative ureteral stent & $6(1.8)$ & $2(4.9)$ & $4(1.4)$ & 0.124 \\
\hline Preoperative PCN & $51(15.4)$ & $8(19.5)$ & $43(14.8)$ & 0.478 \\
\hline Stone size $(\mathrm{mm})$ & $10.1 \pm 4.2$ & $11.7 \pm 3.9$ & $9.9 \pm 4.1$ & 0.004 \\
\hline Operation time (min) & $75.1 \pm 31.6$ & $83.3 \pm 32.2$ & $74.0 \pm 31.3$ & 0.028 \\
\hline Preoperative AGR & $1.64 \pm 0.43$ & $1.46 \pm 0.35$ & $1.66 \pm 0.44$ & 0.003 \\
\hline Preoperative WBC count $\left(10^{3} / \mu \mathrm{L}\right)$ & $6,850 \pm 2,240$ & $6,874 \pm 2,353$ & $6,847 \pm 2,228$ & 0.493 \\
\hline Preoperative serum albumin (g/dL) & $4.5 \pm 0.4$ & $4.2 \pm 0.6$ & $4.5 \pm 0.4$ & 0.001 \\
\hline Preoperative serum globulin (g/dL) & $2.9 \pm 0.5$ & $3.0 \pm 0.6$ & $2.8 \pm 0.4$ & 0.041 \\
\hline Preoperative microscopic hematuria & $142(42.8)$ & $26(63.4)$ & $116(39.9)$ & 0.004 \\
\hline Preoperative hydronephrosis & $111(33.4)$ & $19(46.3)$ & $92(31.6)$ & 0.061 \\
\hline Stone site & & & & 0.144 \\
\hline Renal & $198(59.6)$ & $23(56.1)$ & $175(60.1)$ & \\
\hline Upper & $70(21.1)$ & $10(24.4)$ & $60(20.3)$ & \\
\hline Mid & $16(4.8)$ & $3(7.3)$ & $13(4.5)$ & \\
\hline Low & $48(14.5)$ & $5(12.2)$ & $43(14.8)$ & \\
\hline Preoperative antibiotics cover (day) & $1.5 \pm 2.3$ & $2.5 \pm 4.3$ & $1.4 \pm 1.8$ & 0.003 \\
\hline
\end{tabular}

Values are presented as number (\%) or meantstandard deviation.

AGR, albumin to globulin ratio; fUTI, febrile urinary tract infection; low, low ureter stone; mid, mid ureter stone; PCN, percutaneous nephrostomy; renal, renal stone; RIRS, retrograde intrarenal surgery; upper, upper ureter stone; URS, ureteroscopic lithotripsy; WBC, white blood cell.

ry, sex, preoperative ureteral stenting, preoperative $\mathrm{PCN}$, preoperative WBC counts, and operation sites.

Table 2 shows the results of the multivariate logistic regression analysis for predicting postoperative fUTI. In multivariate logistic regression analysis for the factors that were statistically significant on univariate logistic regression analysis, stone size (OR, 1.1; $95 \%$ CI, 1.003-1.186; $p=0.041$ ), preoperative AGR (OR, 0.4; 95\% CI, $0.170-0.771 ; p=0.008)$, and preoperative pyuria (OR, $5.7 ; 95 \%$ CI, 2.727-12.342; $p<0.001$ ) were found to be significant factors.

According to ROC curves for predicting postoperative fUTI, the optimal cutoff value of AGR was 1.437 (area under the curve [AUC], 0.646; sensitivity, $77.3 \%$; specificity, $76.9 \%$ ) (Table 3), and the optimal cutoff value of stone size was $8.5 \mathrm{~mm}$ (AUC, 0.640; sensitivity, 55.3\%; specificity, 44.7\%) (Table 3).

\section{Discussion}

Advancements in laser technology and ureteroscopy have made lithotripsy less invasive and have yielded a higher stone-free rate with fewer complications. One of the most common postoperative complications is UTI, even when prophylactic antibiotics are adequately administered. Fan et al. [7] found that preoperative pyuria was the most important parameter for UTI, and similarly, our study showed that the presence of pyuria prior to surgery signifi- 
Table 2. Multivariate logistic regression analysis for predicting postoperative febrile urinary tract infection after ureteroscopic lithotripsy and/or retrograde intrarenal surgery

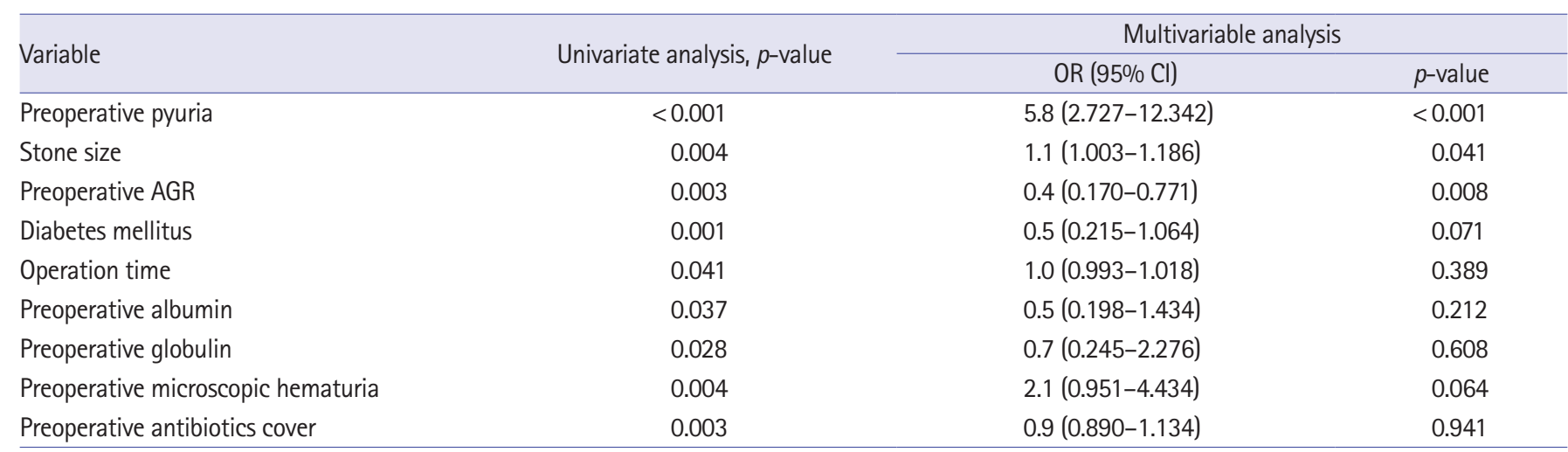

$\mathrm{AGR}$, albumin to globulin ratio; $\mathrm{Cl}$, confidence interval; $\mathrm{OR}$, odds ratio.

Table 3. Receiver operating characteristics curve analysis

\begin{tabular}{lccccccc}
\hline Variable & AUC & SD & $p$-value & $95 \%$ Cl & Cutoff value & Sensitivity (\%) & Specificity (\%) \\
\hline AGR & 0.646 & 0.048 & 0.002 & $0.552-0.741$ & 1.437 & 77.3 & 76.9 \\
Stone size & 0.640 & 0.039 & 0.004 & $0.563-0.717$ & 8.5 & 55.3 & 44.7
\end{tabular}

$A G R$, albumin to globulin ratio; $A U C$, area under the curve; $\mathrm{Cl}$, confidence interval; SD, standard deviation.

cantly contributed to the rate of UTI. Lai and Assimos [8] found that preoperative hydronephrosis was significantly associated with postoperative infection; however, our study showed that hydronephrosis was not a contributing factor to fUTI. In our study, some patients with preoperative hydronephrosis underwent $\mathrm{PCN}$ prior to the operation due to ongoing infection or acute renal failure, which may lower the risk of fUTI in patients with preoperative hydronephrosis.

In our study, the stone size was found to be a risk factor for postoperative fUTI because larger stones may require a longer operation time. Moreover, larger stones might require excessive intrarenal and intraureteral irrigation, which can lead to increased renal pelvic pressure, and the whole sequence would contribute to a higher risk of UTI, which is more likely to increase the absorption of bacteriotoxins and true pathogens [9].

Our results also showed that preoperative AGR is a significant factor that is highly associated with the incidence of postoperative fUTI. Patients with a lower AGR $(<1.437)$ were more susceptible to UTI after URS and RIRS. Previous studies have shown the effectiveness of AGR as a postoperative prognostic factor in patients with different cancers [10-12]. Moreover, Jian et al. [13] and Xun et al. [14] showed the validity of low AGR to predict postoperative fUTI in patients who underwent stone removal operation. Albumin and globulins are major serum proteins that reflect nutritional status and systemic inflammation. Low albumin levels usually reflect malnutrition, which makes one more vulnerable to systemic infections, including UTI. In addition, hypoalbuminemia has been found to lower target organ exposure to antibiotics, which decreases the effectiveness of antibiotics [15]. Non-albumin serum proteins are involved in various inflammatory reactions and consist of proteins such as globulin, C-reactive protein (CRP), and complements $[16,17]$. High globulin components are highly associated with worse prognosis in chronic inflammatory conditions, especially in patients with cancer [18]. High globulin levels reflect the inflammatory and active immune response of a host due to highly accumulated immunoglobulins and CRPs [19]. Hypoalbuminemia and hyperglobulinemia were more prevalent in the fUTI group, but each variable was not found to be a useful predictor of fUTI. Albumin can be influenced by various confounding factors such as stress, illness, hepatic insufficiency, and changes in the volume of body fluids, and this could affect the efficiency and accuracy of albumin as a prognostic marker [20]. Previous studies have proposed that AGR provides an assessment of albumin and globulin together, which could reflect the body's nutritional status and inflammatory states comprehensively, and this increases its value as a novel biochemical index $[20,21]$. Thus, patients with a lower AGR could be more susceptible to acute inflammatory conditions (especially fUTI) after surgery.

Our study had some limitations. First, our study had the potential for selection bias due to the retrospective nature of the study design. Second, more inflammatory markers such as NLR, PLR, $\mathrm{CRP}$, and erythrocyte sedimentation rate were not estimated in 
our study due to the retrospective nature of our study design. Finally, because our study population included patients from only one institution, ethnic and geographic variations in stone disease were not reflected in the study population.

Predicting postoperative fUTI after URS and RIRS is important because this complication can be life-threatening and can increase the cost of healthcare, mortality of patients, and hospital stay. Evaluation of preoperative AGR is cost-effective and reliable for predicting postoperative fUTI. According to our study, patients with preoperative pyuria, AGR below 1.437, or stone size greater than $8.5 \mathrm{~mm}$ should be monitored closely after surgery. Monitoring vital signs and giving careful attention to clinical symptoms would be necessary to manage postoperative fUTI. Further large-scale prospective studies are required to validate the efficacy of AGR in predicting postoperative fUTI.

\section{Notes}

\section{Ethical statements}

This was a retrospective study using personal medical records, and informed consent was waived. The study was approved by the Institutional Review Board (IRB) of Kyungpook National University Hospital (IRB No: 2020-10-009-001).

\section{Conflicts of interest}

No potential conflict of interest relevant to this article was reported.

\section{Funding}

This research was supported by the Basic Science Research Program through the National Research Foundation of Korea (NRF) \& funded by the Korean government (MSIT) (2019R1F1A1044473) (2019R1H1A1079839) (2020R1I1A3071568).

\section{Author contributions}

Conceptualization: SYY, YSH, ESY; Data curation: DJP, KM; Investigation: SYY, THK; Formal analysis: DJP, KM; Funding acquisition: YSH, BSK; Validation: ESY; Writing-original draft: SYY; Writing-review \& editing: JWC, HTK, ESY.

\section{ORCID}

Seung Yun Yi, https://orcid.org/0000-0003-4736-8198

Dong Jin Park, https://orcid.org/0000-0003-1667-2146

Kyungchan Min, https://orcid.org/0000-0001-5238-6265

Jae-Wook Chung, https://orcid.org/0000-0002-1055-2357

Yun-Sok Ha, https://orcid.org/0000-0003-3732-9814

Bum Soo Kim, https://orcid.org/0000-0002-4873-3049
Hyun Tae Kim, https://orcid.org/0000-0002-4730-3776

Tae-Hwan Kim, https://orcid.org/0000-0003-4924-4826

Eun Sang Yoo, https://orcid.org/0000-0002-7442-6886

\section{References}

1. Baseskioglu B. The prevalence of urinary tract infection following flexible ureterenoscopy and the associated risk factors. Urol J 2019;16:439-42.

2. Senocak C, Ozcan C, Sahin T, Yilmaz G, Ozyuvali E, Sarikaya S, et al. Risk factors of infectious complications after flexible uretero-renoscopy with laser lithotripsy. Urol J 2018;15:158-63.

3. Knopf HJ, GraffHJ, Schulze H. Perioperative antibiotic prophylaxis in ureteroscopic stone removal. Eur Urol 2003;44:115-8.

4. Cetinkaya M, Buldu I, Kurt O, Inan R. Platelet-to-lymphocyte ratio: a new factor for predicting systemic inflammatory response syndrome after percutaneous nephrolithotomy. Urol J 2017;14:4089-93.

5. Sen V, Bozkurt IH, Aydogdu O, Yonguc T, Yarimoglu S, Sen P, et al. Significance of preoperative neutrophil-lymphocyte count ratio on predicting postoperative sepsis after percutaneous nephrolithotomy. Kaohsiung J Med Sci 2016;32:507-13.

6. He J, Pan H, Liang W, Xiao D, Chen X, Guo M, et al. Prognostic effect of albumin-to-globulin ratio in patients with solid tumors: a systematic review and meta-analysis. J Cancer 2017;8:400210.

7. Fan S, Gong B, Hao Z, Zhang L, Zhou J, Zhang Y, et al. Risk factors of infectious complications following flexible ureteroscope with a holmium laser: a retrospective study. Int J Clin Exp Med 2015;8:11252-9.

8. Lai WS, Assimos D. Factors associated with postoperative infection after percutaneous nephrolithotomy. Rev Urol 2018;20:711.

9. Li T, Sun XZ, Lai DH, Li X, He YZ. Fever and systemic inflammatory response syndrome after retrograde intrarenal surgery: Risk factors and predictive model. Kaohsiung J Med Sci 2018; 34:400-8.

10. Azab BN, Bhatt VR, Vonfrolio S, Bachir R, Rubinshteyn V, Alkaied $\mathrm{H}$, et al. Value of the pretreatment albumin to globulin ratio in predicting long-term mortality in breast cancer patients. Am J Surg 2013;206:764-70.

11. Du XJ, Tang LL, Mao YP, Sun Y, Zeng MS, Kang TB, et al. The pretreatment albumin to globulin ratio has predictive value for long-term mortality in nasopharyngeal carcinoma. PLoS One 2014;9:e94473.

12. Duran AO, Inanc M, Karaca H, Dogan I, Berk V, Bozkurt O, et al. Albumin-globulin ratio for prediction of long-term mortality 
in lung adenocarcinoma patients. Asian Pac J Cancer Prev 2014;15:6449-53.

13. Jian ZY, Ma YC, Liu R, Li H, Wang K. Preoperative positive urine nitrite and albumin-globulin ratio are independent risk factors for predicting postoperative fever after retrograde Intrarenal surgery based on a retrospective cohort. BMC Urol 2020;20:50.

14. Xun Y, Yang Y, Yu X, Li C, Lu J, Wang S. A preoperative nomogram for sepsis in percutaneous nephrolithotomy treating solitary, unilateral and proximal ureteral stones. PeerJ 2020;8:e9435.

15. Ulldemolins M, Roberts JA, Rello J, Paterson DL, Lipman J. The effects of hypoalbuminaemia on optimizing antibacterial dosing in critically ill patients. Clin Pharmacokinet 2011;50:99110.

16. Codina Cazador A, Salvá Lacombe JA, Fernández-Llamazares Rodríguez J, Ruiz Feliu B, Codina Barreras A, Moreno Aguado V. Immunoglobulins and the complement system in colorectal cancer. Rev Esp Enferm Apar Dig 1989;75:143-8.
17. Cohen MH, Makuch R, Johnston-Early A, Ihde DC, Bunn PA Jr, Fossieck BE Jr, et al. Laboratory parameters as an alternative to performance status in prognostic stratification of patients with small cell lung cancer. Cancer Treat Rep 1981;65:187-95.

18. Zhang W, Zhangyuan G, Wang F, Zhang H, Yu D, Wang J, et al. High preoperative serum globulin in hepatocellular carcinoma is a risk factor for poor survival. J Cancer 2019;10:3494-500.

19. Du XJ, Tang LL, Mao YP, Guo R, Sun Y, Lin AH, et al. Circulating EBV DNA, globulin and nodal size predict distant metastasis after intensity-modulated radiotherapy in stage II nasopharyngeal carcinoma. J Cancer 2016;7:664-70.

20. Zhou T, He X, Fang W, Zhan J, Hong S, Qin T, et al. Pretreatment albumin/globulin ratio predicts the prognosis for smallcell lung cancer. Medicine (Baltimore) 2016;95:e3097.

21. Zhang Y, Wang L, Lin S, Wang R. Preoperative albumin-to-globulin ratio as a significant prognostic indicator in urologic cancers: a meta-analysis. Cancer Manag Res 2018;10:4695-708. 